\title{
Desenvolvimento de um Laboratório Virtual para Capacitação Tecnológica à Distância em Proteção de Sistemas Elétricos
}

\author{
Carlos Henrique de Castro Silva - DEE/UFC - Universidade Federal do Ceará, \\ carloshenriquecs@gmail.com \\ Raimundo Furtado Sampaio - DEE/UFC - Universidade Federal do Ceará, \\ rfurtado@dee.ufc.br \\ Ruth Pastôra Saraiva Leão - DEE/UFC - Universidade Federal do Ceará, \\ rleao@dee.ufc.br \\ Giovanni Cordeiro Barroso - Universidade Federal do Ceará, \\ gcb@fisica.ufc.br \\ José Marques Soares - DETI/CT - Universidade Federal do Ceará, \\ marques@ufc.br
}

\begin{abstract}
Resumo: A necessidade por profissionais cada vez mais qualificados, bem como o custo associado à liberação de funcionários para participar destes cursos, forçaram a pesquisa de novas formas de treinamento que possibilitassem um bom resultado profissional e racionalizassem a disposição de recursos humanos. Para atender essa demanda, foi desenvolvido um Laboratório Virtual para Capacitação Tecnológica presencial e à Distância em Proteção de Sistemas Elétricos. O laboratório virtual, denominado Sistema Simulador para Treinamento Presencial e a Distância da Proteção de Sistemas Elétricos (STOP), pode ser aplicado em treinamento presencial e à distância e está integrado ao Moodle, enriquecendo o ambiente com uma ferramenta capaz de simular situações reais que pode proporcionar um ensino-aprendizado mais eficaz.
\end{abstract}

Palavras-chave: proteção de sistemas elétricos, simulador de operação de subestação, educação à distância, funções e ajuste de proteção.

\section{Virtual Laboratory for Technology Classroom Training and Distance Classes on Eletric Systems Protection}

\begin{abstract}
The need for each time more qualified professionals, as well as the cost associated with the release of staff to attend these courses, forced the search for new forms of training that would enable nice professional results and rationalization of human resources provision. To attend this demand, we developed a Virtual Laboratory for Technology Classroom Training and Distance Classes on Eletric Systems Protection. The Virtual Laboratory, called Simulator System for Classroom Training and Distance Classes on Eletric Systems Protection (STOP), can be applied in both classroom and distance training, and is integrated to the Moodle, enriching the environment with a tool capable of simulating real situations can provide a more effective teaching-learning process.
\end{abstract}

Keywords: protection of electrical systems, substation simulator operation, distance education, protection and adjustment functions.

\section{Introdução}

A constante evolução tecnológica dos sistemas de controle e proteção tem proporcionado melhoria na segurança e confiabilidade dos sistemas de potência. Em 
contrapartida, para acompanhar a velocidade das mudanças, garantindo a segurança e a confiabilidade dos sistemas elétricos, as empresas necessitam de profissionais qualificados, devendo para isto investir em capacitação e atualização profissional continuada dos profissionais próprios e terceirizados. No entanto, na conjuntura atual, as empresas se deparam com a dificuldade de liberação de pessoal para participar de treinamentos. Estes fatores têm conduzido à busca de soluções que venham a atender à demanda das empresas, mantendo a qualidade do processo de formação continuada (Bezerra, 2008).

O desenvolvimento na área de Tecnologia de Informação e Computação tem permitido que novas formas de treinamento sejam desenvolvidas, com recursos necessários a um treinamento com qualidade nas modalidades presencial, semipresencial e à distância.

Os Ambientes Virtuais de Aprendizagem (AVAs) oferecem um conjunto de ferramentas para a realização de cursos nestas várias modalidades, que, combinadas ou isoladas, permitem a melhor convivência de aprendizes e formadores com as dimensões críticas impostas pelo distanciamento físico e temporal deste tipo de ambiente.

No caso da Educação Tecnológica e Profissional a Distância (ETPD), o uso de ferramentas virtuais, como simuladores, tem sido uma alternativa de redução de custos para as empresas e instituições de ensino.

No contexto do ETPD, é desejável que os AVAs possam ser complementados por simuladores ou Laboratórios Virtuais, o que não ocorre com frequência na prática.

Vários são os ambientes virtuais de aprendizagem existentes nas diversas áreas do conhecimento. Entretanto, estes ambientes geralmente não oferecem acesso a simuladores externos, desenvolvidos em outras linguagens de programação.

A literatura, entretanto, aponta algumas experiências realizadas com simuladores desenvolvidos especificamente para treinamento à distância. $\mathrm{O}$ simulador STOP é a evolução de um simulador desenvolvido utilizando a linguagem DELPHI, STPO $^{[1]}$ (Sistema Simulador para Treinamento Presencial e a Distância de Operação da Proteção de Sistemas Elétricos) e que se encontra integrado ao TelEduc. É relatada na literatura a experiência da Companhia Energética de São Paulo (CESP) no treinamento à distância de operadores (Fuzeto, 2003), assim como a experiência da Companhia de Transmissão de Energia Elétrica Paulista (CTEEP) (Teixeira et al., 2003) com treinamento de operadores de subestações e despachantes utilizando simulador de treinamento para operação de sistemas elétricos.

O texto está disposto da seguinte forma: na seção 2, abordamos a motivação para o desenvolvimento da ferramenta e a integração ao Moodle; na seção 3, é exposto o simulador e suas principais funções; na seção 4; descreve aspectos sobre a implantação, validação e resultados da integração; na seção 5; são apresentadas as considerações finais e perspectivas para desenvolvimento futuro do simulador.

\section{Integração da ferramenta ao Moodle}

Um problema que pode se apresentar com frequência para desenvolvedores que precisem agregar ferramentas previamente existentes aos AVAs é a incompatibilidade de tecnologias. Alguns simuladores são desenvolvidos em C, Delphi ou outras linguagens, enquanto que os AVAs são, em sua grande maioria, baseados em tecnologia Web (Soares et al., 2006). 


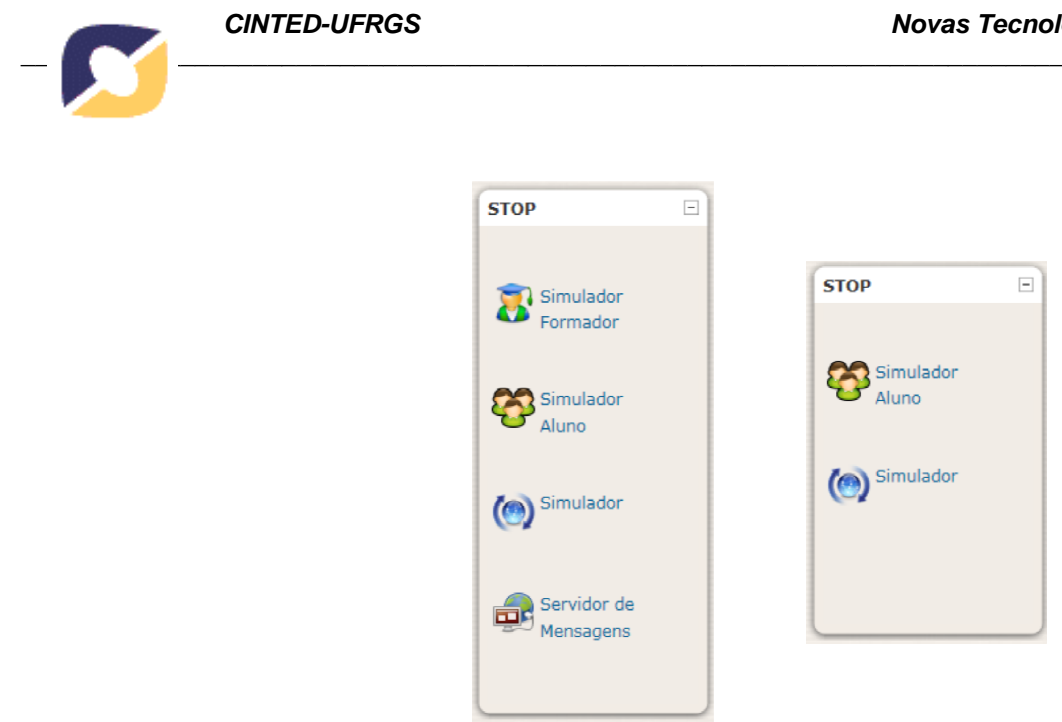

Figura 1: Bloco STOP integrado ao Moodle

A integração da ferramenta ao Moodle é feita através de um bloco, denominado STOP (Figura 1). A principal funcionalidade do bloco desenvolvido é permitir ao professor/tutor acesso às ferramentas de configuração e controle do ambiente (Simulador Formador e Servidor de Mensagens) e, aos alunos, somente o acesso ao Simulador Aluno e Simulador.

Os elementos do bloco são:

- Simulador Formador: Permite acesso à ferramenta de acesso remoto, disponibilizado apenas para o professor/tutor (Figura 2);

- Simulador Aluno: Permite acesso à ferramenta de acesso remoto, disponibilizado para o professor/tutor e aluno (Figura 2);

- Simulador: Versão do software simulador para utilização local utilizando Java Web Start, que permite fazer download de aplicativos Java da web.

- Servidor de Mensagens: Permite acesso à configuração

A ferramenta desenvolvida foi projetada para que se pudesse acessar o simulador remotamente usando o sistema VNC (Virtual Network Computing). O VNC é um sistema cliente servidor que permite o acesso à interface gráfica de um computador remoto, permitindo ainda o compartilhamento deste acesso. A ferramenta desenvolvida agrega funcionalidades para a gestão do acesso ao computador remoto, além de integrar mecanismos de comunicação e colaboração, como pode ser visto na Figura 2.

\subsection{Interface de acesso ao Simulador}

O STOP foi desenvolvido em Java, integrando as classes de um cliente TightVNC, estendendo-as para incluir algumas adaptações. Durante o início do projeto foi necessário escolher entre manter a arquitetura utilizando o $\mathrm{VNC}$, ou definir uma nova arquitetura sem a necessidade de utilização do VNC, no desenvolvimento do software em Java. Optou-se pela arquitetura utilizando o VNC em virtude da possibilidade de utilização da ferramenta em cursos utilizando softwares desenvolvidos para Windows, visto que grande parte dos softwares de configuração e parametrização dos relés são desenvolvidos exclusivamente para o sistema operacional Windows.

A interface desta ferramenta para o formador (professor/tutor) é apresentada na Figura 2. A interface do aluno difere apenas do Painel de Liberação de Recursos, que, no caso do aluno, se chama Painel de Solicitação de Recurso, apresentado na Figura 3. Os elementos da Interface são descritos em seguida. 


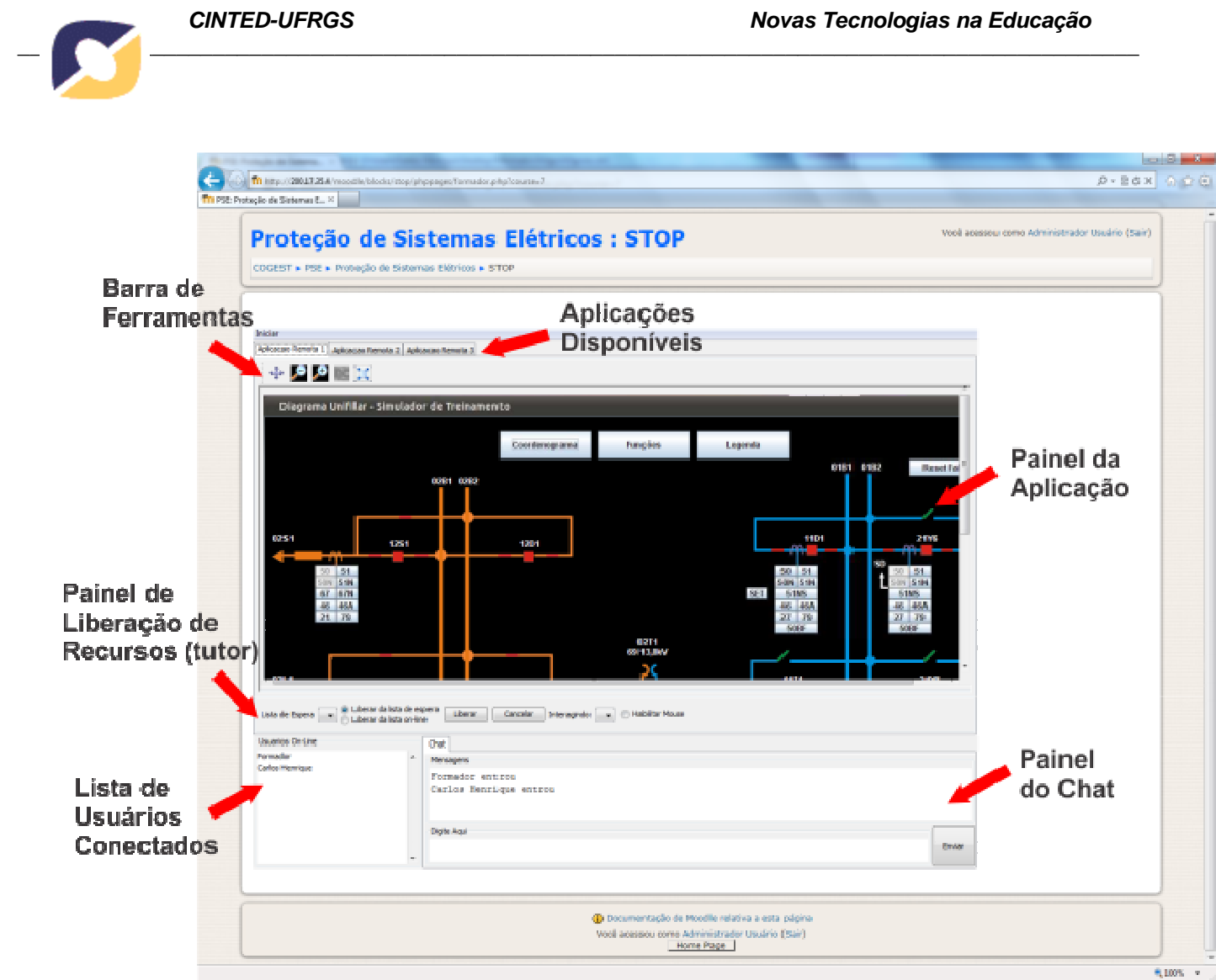

Figura 2: Interface da ferramenta de acesso remoto

Os elementos da Interface são descritos a seguir:

- Aplicações disponíveis: Ambiente gráfico compartilhado em que cada aba disponível representa uma "sala de aula virtual", podendo ser configuradas para acesso remoto pelos alunos.

- Painel da Aplicação: Corresponde à interface do computador remoto, que executa a aplicação compartilhada por tutores e alunos. $\mathrm{O}$ acesso a esta região é mediado pelo tutor, podendo ele atribuir ou retirar o direito de acesso a um único aluno por vez.

- Barra de Ferramentas: Contém os botões que atuam sobre o Painel da Aplicação, com opções de pan, de zoom in e de zoom out.

- Painel do Chat - Área onde podem ser trocadas mensagens entre alunos e tutor durante a colaboração. Quando o Painel da Aplicação é apresentado em tela cheia, o chat é transportado para uma janela suspensa.

- Lista de Usuários Conectados: Nesta parte da interface pode-se ver a lista de usuários conectados à aplicação.

- Painel de Liberação de Recursos: Neste painel, o tutor observa os pedidos de liberação de acesso à aplicação remota. Quando o aluno requisita acesso, seu nome é apresentado na lista de usuários que estão aguardando a liberação da ferramenta. Na caixa de texto status, é apresentado o nome do aluno que detém o direito de acesso no momento. Este painel não existe na interface do aluno, sendo substituído pelo Painel de Solicitação de Recursos, como mostrado na Figura 3.

Lista de Espera Alinos - Interagin Cancelar Interagndo Carlos Herrique.

Figura 3: Painel de solicitação de recursos (aluno) 
A interface apresenta flexibilidade suficiente para a agregação de novos componentes. Pode-se trabalhar, por exemplo, com múltiplas instâncias do VNC na mesma interface, permitindo o acesso simultâneo a diferentes aplicações remotas em localizações distintas. Em qualquer situação, o usuário escolhe qual a aplicação que deve ser colocada em evidência no Painel da Aplicação.

\subsection{Arquitetura}

Os componentes da arquitetura utilizada são distribuídos como ilustrado na Figura 4. Os clientes, que são os tutores e alunos, usando um navegador, acessam o Servidor $W E B$ em que está instalado o Moodle. Neste servidor, além do próprio Servidor $W e b$, encontram-se em execução os seguintes módulos: um Servidor de Mensagens, responsável pela comunicação por texto, um Módulo de Coordenação, responsável pelo controle de acesso à aplicação compartilhada, e um Refletor VNC, que faz a intermediação da comunicação com a aplicação compartilhada (proxy).

No Servidor de Aplicações, encontram-se em execução um Servidor VNC e a Aplicação Compartilhada. Em nossa arquitetura, o Servidor de Aplicações é situado na mesma rede local que o Servidor Web.

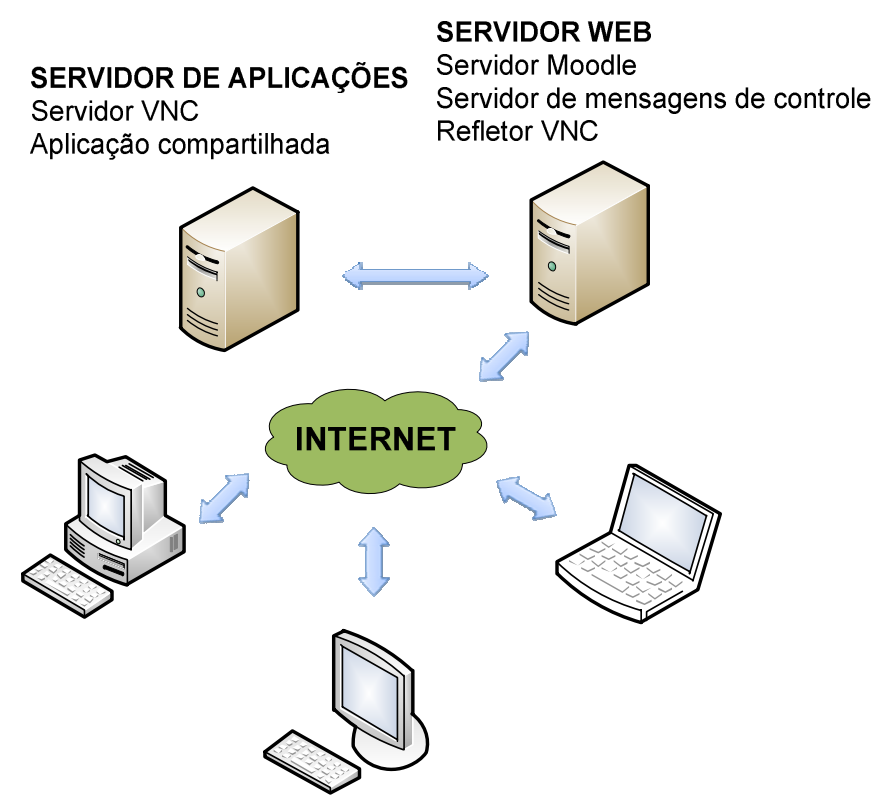

Figura 4: Distribuição dos componentes da arquitetura

O Servidor de Mensagens de Controle é um servlet que pode ser acionado remotamente e permite a configuração dos IPs das portas, e das senhas usadas pelo ambiente. A interface para esta configuração é construída em JSP (Java Server Pages), conforme mostrada na (Figura 5).

O Servidor de Aplicações e o Servidor Web podem pertencer a redes diferentes, desde que o Servidor de Aplicações possua um IP válido e esteja executando uma aplicação VNCServer. Basta configurar os endereços, porta e senha como parâmetros para o Servidor de Mensagens de Controle (Figura 5). 


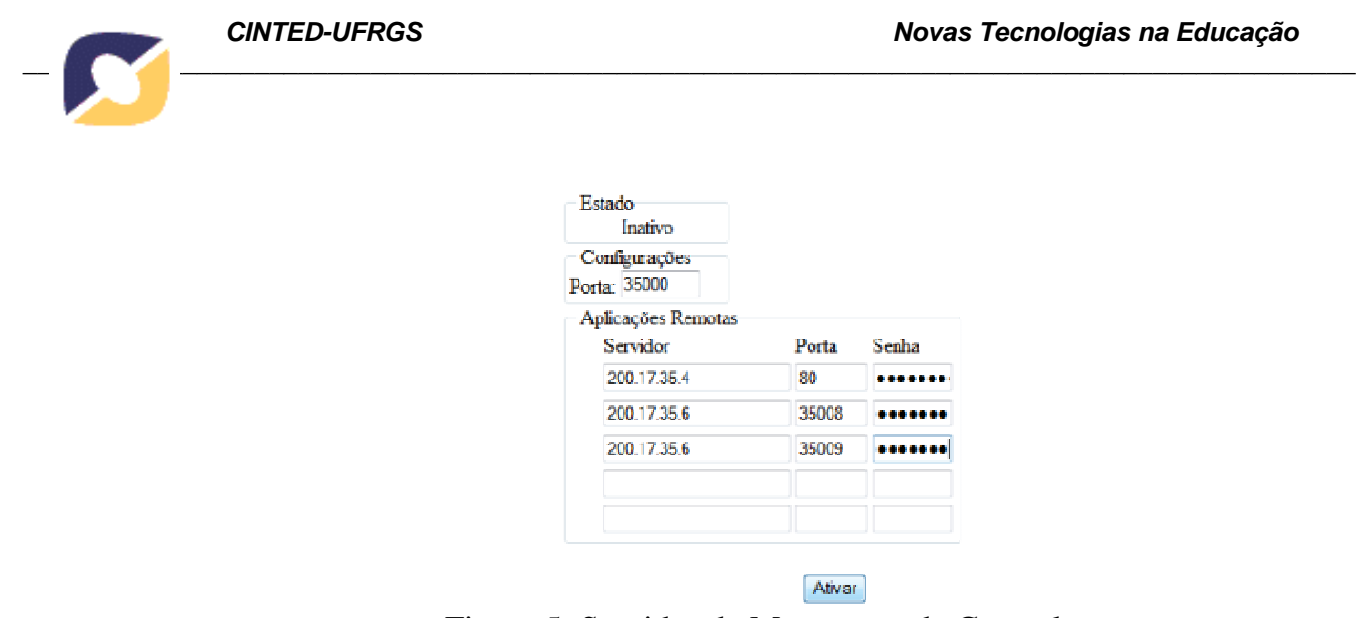

Figura 5: Servidor de Mensagens de Controle

Toda a dinâmica de uma sessão síncrona entre tutor e alunos pode ser gravada na maquina do Servidor WEB. Para isso, utiliza-se o programa vnc2flv, uma ferramenta de gravação de tela para Linux, Windows ou Mac. Ele captura uma sessão de desktop VNC (sua própria tela ou de um computador remoto) e salva como um arquivo Flash Vídeo (FLV) para posterior consulta dos alunos, conforme ilustrado na Figura 6.

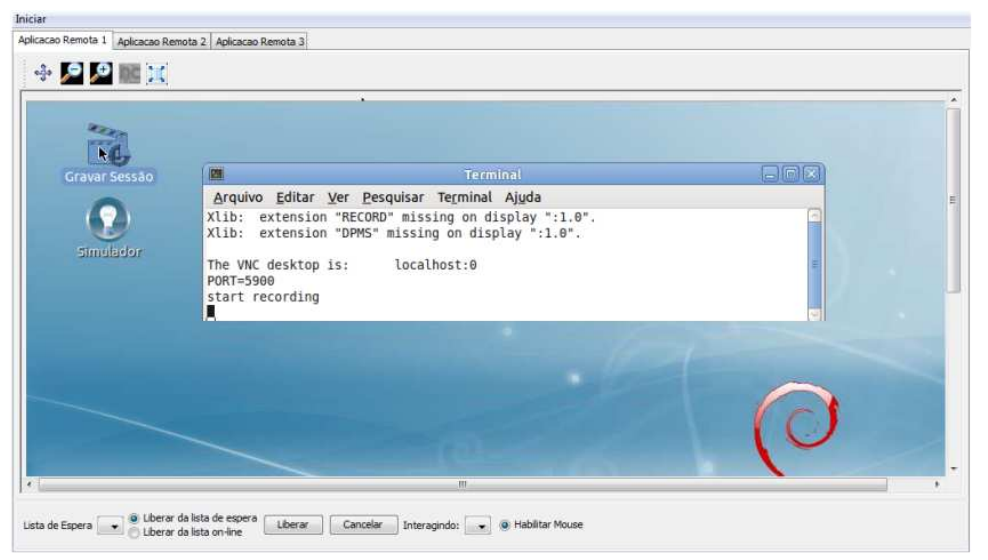

Figura 6: Gravação de uma sessão utilizando vnc2flv

\section{O Simulador do Sistema Elétrico}

O (STOP) tem como objetivo permitir a capacitação e treinamento de pessoal e estudantes do setor elétrico através do ensino presencial e à distância via internet.

O STOP é um software composto de diagramas unifilares sistêmicos e específicos com a representação dos principais componentes do sistema elétrico de proteção, tais como disjuntores, religadores, transformadores e relés. No simulador, o usuário pode configurar o sistema utilizando dados de equipamentos reais utilizados no sistema elétrico de potência, bem como simular faltas e reconfigurar o sistema, fazendo ajustes de relés para coordenação e redefinição de parâmetros. A tela inicial do STOP é mostrada na Figura 11. Nela é apresentado o diagrama unifilar da subestação com seus respectivos equipamentos de proteção e operação que podem ser configurados com valores reais de equipamentos existentes no mercado e testados através das simulações.

As principais funções que o simulador dispõe para simulações no sistema elétrico, necessárias à consolidação e validação dos conhecimentos dos alunos, são:

Especificação da relação nominal dos transformadores de corrente (RTC), permitindo ao aluno a escolha de equipamentos comercialmente utilizados nos sistemas elétricos (Figura 7). 


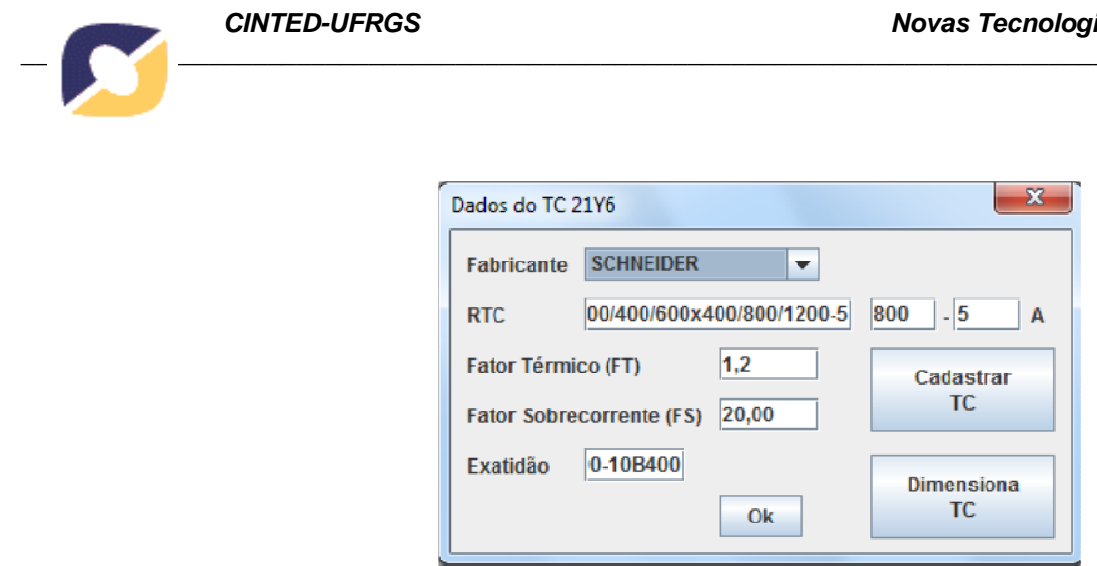

Figura 7: Especificação dos TCs

Verificação da correção do dimensionamento do equipamento utilizado, clicando no botão Dimensiona TC. Nesse caso, será exibida uma mensagem informando ao aluno se a RTC utilizada é válida ou não, baseada no critério de carga máxima e no critério de curto-circuito (Figura 8). Se o aluno escolher uma RTC não disponível no fabricante escolhido, é exibida a mensagem de erro RTC inválida (Figura 8).

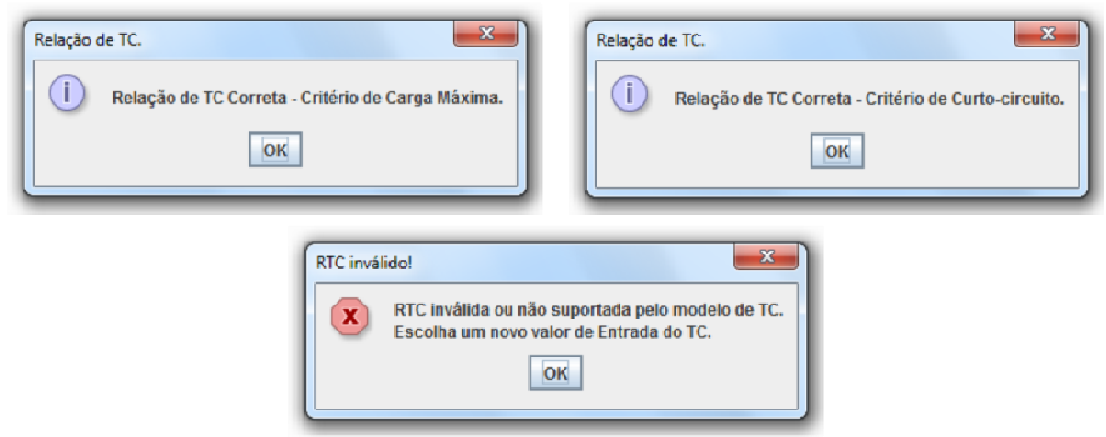

Figura 8: Verificação do dimensionamento do TC

Implantação de critérios de ajuste e métodos de cálculo de cada função de proteção (50 - Sobrecorrente instantânea de fase (Figura 10), 51 - Sobrecorrente temporizada de fase (Figura 9), 50N - Sobrecorrente instantânea de neutro, 51N Sobrecorrente temporizada de neutro, 79 - Função de Religamento, 50BF - Função falha de disjuntor, 51NS - Neutro sensível).

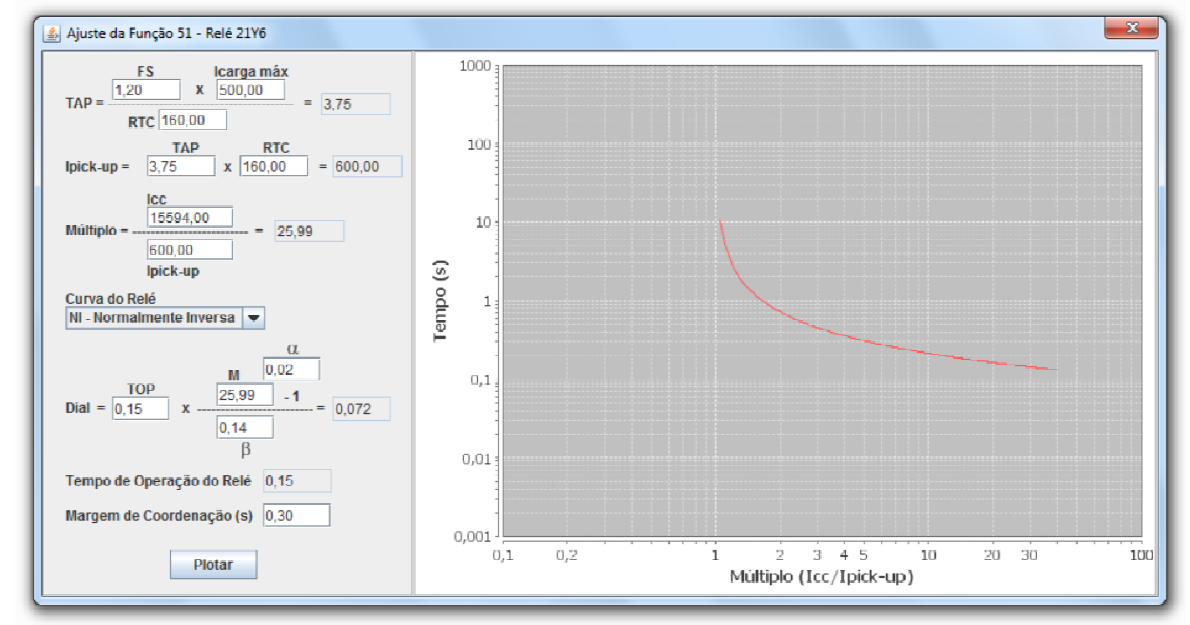

Figura 9: Configuração do ajuste da função 51

Geração de faltas em alimentadores com efeitos visuais indicativos de curtoscircuitos, simulação da atuação das funções de proteção, com a abertura dos disjuntores associados de acordo com o local e o tipo de falta (Figura 11). 


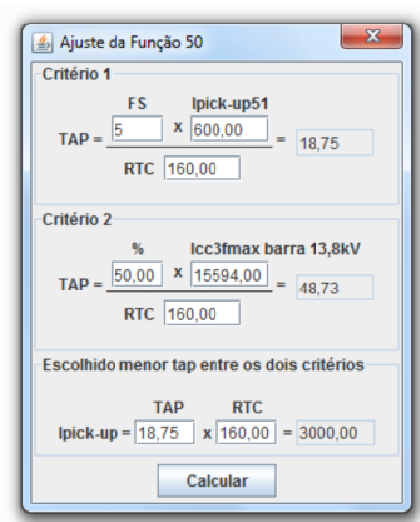

Figura 10: Configuração do ajuste da função 50

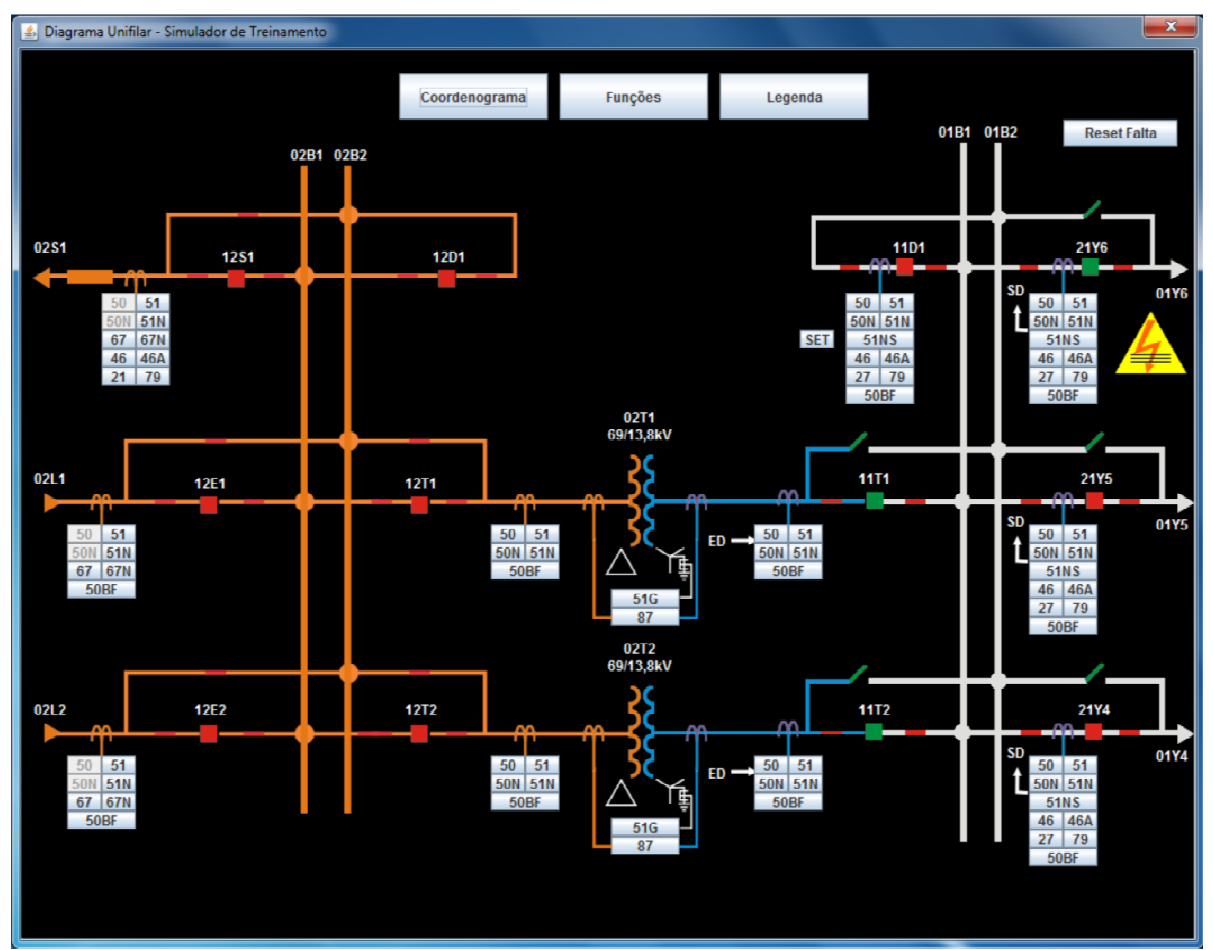

Figura 11: Indica a ação dos dispositivos de proteção, mostrando o local e o tipo de curto-circuito

Geração de coordenograma das proteções, após a definiç̧ão das curvas e ajuste dos relés de sobrecorrente, gerar um coordenograma para verificar se as proteções estão coordenadas (Figura 12).

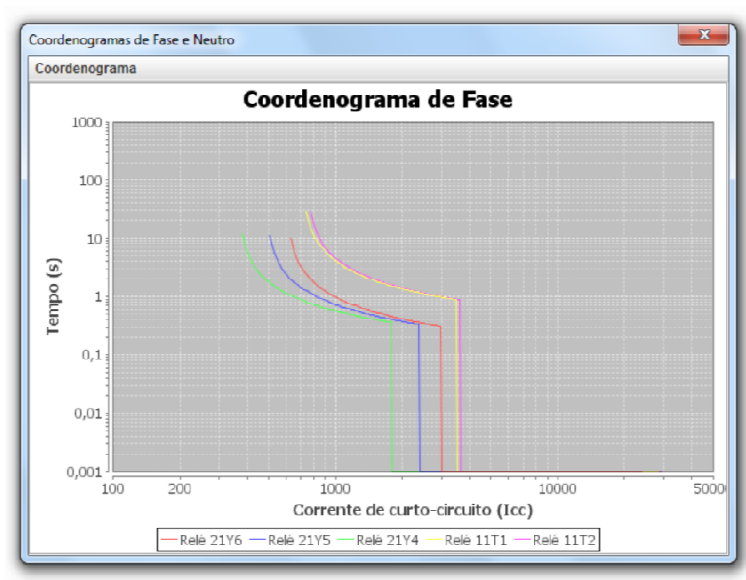

Figura 12: Coordenograma de fase 


\section{Implantação, Resultados e Validação}

A validação dos resultados obtidos com o software de simulação STOP foram feitas através do software MathCad, um software de matemática e engenharia para cálculos numéricos e simbólicos. O software desenvolvido integrado ao ambiente virtual de aprendizagem Moodle foi implantado em servidores do COGEST e disponibilizado através do endereço http://cogest.fisica.ufc.br/moodle. A primeira fase do projeto foi o desenvolvimento do simulador e integração do mesmo junto ao Moodle. A próxima etapa será a experimentação da ferramenta junto aos alunos da disciplina de Proteção de Sistemas Elétricos do curso de Engenharia Elétrica da Universidade Federal do Ceará e avaliação do mesmo junto aos alunos das disciplinas, de forma a obter resultados relevantes para o aprendizado, comprovando a eficácia do simulador como uma ferramenta de apoio ao ensino de proteção de sistemas elétricos.

\section{Considerações Finais e Perspectivas}

Neste trabalho foi apresentado o desenvolvimento de um sistema para capacitação presencial e à distância de profissionais e estudantes na área de proteção e operação de sistemas elétricos.

Além do embasamento teórico disponibilizado no Moodle, com atividades de exercício, avaliação e questionários, o sistema de capacitação oferece um simulador para treinamento da proteção e operação. O STOP é uma ferramenta capaz de simular situações reais, proporcionando um ambiente de ensino e aprendizagem à distância eficaz.

O STOP integrado ao Moodle, como apresentado neste trabalho, além de viabilizar o acesso a uma ferramenta de treinamento específico, pode ser utilizado em cenários externos aos ambientes virtuais de aprendizagem, oferecendo um suporte relevante à percepção mútua das ações efetuadas entre usuários distantes, o que é estimulado não só pela troca de mensagens síncronas através do chat, mas pelo acompanhamento da dinâmica do compartilhamento de um mesmo aplicativo, identificando o status de cada participante e permitindo a análise da interação do usuário ativo sobre a aplicação compartilhada a cada instante. As interações com o simulador, integradas aos mecanismos de comunicação fornecidos pela ferramenta e aos demais recursos oferecidos pelo AVA, permitem ao aprendiz aplicar, transformar e buscar outras informações, construindo seu conhecimento. são eles:

Novos recursos estão sendo adicionados para aprimoramento da ferramenta,

- Parametrização dos relés a partir do simulador;

- Acesso ao aluno na criação e configuração de uma subestação, de forma dinâmica, criando diferentes topologias de subestação;

- Geração da planilha de Ordem de Ajuste das Proteções (OAP) e da planilha de dados dos equipamentos a partir dos dados do STOP;

- Captura dos registros dos relés via STOP;

- Integração de ferramentas de áudio e vídeo ao ambiente virtual. 


\section{Referências}

BEZERRA, H., Sistema Simulador para Treinamento Presencial e a Distância de Operação da Proteção de Sistemas Elétricos, Universidade Federal do Ceará - UFC, 2008, 122p.

FUZETO, J. Treinamento à distância de operadores com infotecnologias e recursos da web na era da cibercultura - A experiência da CESP. SNPTEE - Seminário Nacional De Produção e Transmissão de Energia, XV., Uberlândia, Minas Gerais, 2003.

SOARES, J. M. LEITE, F. R. MATTOS, C. L. C. BARROSO, G. C. SAMPAIO, R. F. LEÃO, R. P. S. Acesso Remoto e Compartilhamento de Simuladores através de Ambientes Virtuais de Aprendizagem. In: Simpósio Brasileiro de Informática na Educação, XVII., 2006, Brasília. p. 308-317.

TEIXEIRA, E. C., LOPEZ, R. M., Ruy, O. L., SANTOS, L. A., FREITAS, J. A. S. Treinamento de Operadores de Subestações e Despachantes Utilizando Simulador de Treinamento para Operação de Sistemas Elétricos. SIMPASE - Simpósio de Automação de Sistemas Elétricos, V., Recife, 2003. 